\title{
The angular momentum of condensations within elephant trunks
}

\author{
V. Lora ${ }^{1}$, A. C. Raga ${ }^{2}$, and A. Esquivel ${ }^{2}$ \\ 1 Instituto de Astronomía, Universidad Nacional Autónoma de México, Ap. 70-468, 04510, D.F México \\ 2 Instituto de Ciencias Nucleares, Universidad Nacional Autónoma de México, Ap. 70-543, 04510, D.F México \\ e-mail: raga@nucleares.unam.mx
}

Received 31 March 2009 / Accepted 22 May 2009

\section{ABSTRACT}

\begin{abstract}
Aims. The radiation from newly born stars photoevaporates their parental neutral cloud, leading to the formation of dense clumps that will eventually form stars.

Methods. We present 3D simulations of the interaction of a neutral cloud with an external ionising radiation field, and compute the angular momenta of these collapsing clumps.

Results. The angular momenta of these collapsing clumps show that they have preferential orientations mostly perpendicular to the direction of the incident ionising photon field. Therefore, the axes of the jet systems that will be eventually ejected (from the star + accretion disk systems that will form) will be oriented approximately perpendicular to the direction to the photoionising source.
\end{abstract}

Key words. ISM: kinematics and dynamics - ISM: clouds - ISM: HII regions - stars: formation

\section{Introduction}

The radiation from newly born stars photoionises and erodes the parental cloud, producing structures such as the so-called elephant trunks. At the head of an elephant trunk, the interaction of the shock (driven by the photoevaporation process) with previously existing density perturbations leads to the formation of dense clumps. Some of these clumps might have enough mass to be self-gravitating, and will eventually form young stars that eject bipolar outflows. We describe observed examples of this kind of configuration.

Bally \& Reipurth (2003) discovered HH objects in the molecular cloud associated with the Pelican Nebula, including $\mathrm{HH}$ 555. This outflow emerges from the tip of a long elephant trunk, providing direct evidence of ongoing star formation in this region. The outflow axis of HH 555 is approximately perpendicular to the elephant trunk, which is aligned with the direction to the photoionising source.

Another example of this kind of configuration is the HH 666 outflow in the Carina nebula. HH 666 also emerges from close to the tip of an elephant trunk, and its axis is almost perpendicular to the direction towards $\eta$ Carinae (Smith et al. 2004). An HST image of this region (Bally et al. 2007), shows a second jet emerging from a nearby elephant trunk, with a direction almost parallel to the HH 666 outflow.

A final example is provided by $\mathrm{HH} 333$. This jet emerges from the tip of an elephant trunk within the complex Trifid nebula (M20). It is a single-sided jet with measured radial velocities (Rosado et al. 1999) and proper motions (Yusef-Zadeh et al. 2005) that indicate it has the kinematical properties of a standard $\mathrm{HH}$ jet. Again $\mathrm{HH} 333$ has an outflow direction approximately perpendicular to the direction to the ionising source.

Reach et al. (2009) presented observations of the elephant trunk of globule IC 1396A. They detected outflow activity from a number of young stars in the region. However, it is impossible to determine the outflow axes from these observations.

Even though the number of four outflows (two in the $\mathrm{HH} 666$ region, see above) observed to be emerging from tips of elephant trunks is quite small, their alignment approximately perpendicular to the direction to the ionising photon source might be indicative of a systematic alignment. This alignment implies that the angular momenta of the low mass star+disk systems producing outflows from stellar sources in the tip of elephant trunks are more or less perpendicular to the direction of the ionising photon field (produced by the massive stars giving rise to the photoionised nebulae and elephant trunk structures). These angular momenta presumably preserve the direction of the rotation axes of the dense clumps that collapsed to form the outflow sources.

In the present paper, we explore the interaction between an ionising photon field and an environment with density perturbations. This interaction produces elongated structures reminiscent of elephant trunks, with dense, embedded clumps. In particular, we focus on whether or not these dense clumps have angular momenta preferentially oriented perpendicular to the direction towards the photoionising source.

Mellema et al. (2006) carried out 3D, radiation gasdynamic simulations of an H II region expanding in an ISM with powerlaw density perturbations. They find that this configuration naturally leads to the formation of dense, radially elongated structures, which resemble elephant trunks. Gahm et al. (2006) also study the role of magnetic fields in the formation of elephant trunks. Finally, Gritschneder et al. (2009) carried out a simulation of an initially plane ionising front travelling into a structured medium.

Our work emulates the approach of Mellema et al. (2006) and Gritschneder et al. (2009). We focus on a small region at the edge of an expanding H II region, and carry out a 3D radiation gasdynamic simulation (including the self-gravity of the gas) of the formation of a dense, neutral structure. We then identify high density clumps within this "elephant trunk", and compute their angular momenta. Finally, we study the mass distribution of the clumps, and the distributions of the orientation and magnitude of their angular momenta. 
The paper is organized as follows. In Sect. 2, we describe the gasdynamic code and the parameters used for the numerical simulation. The results from the simulation and the clump statistics are presented in Sect. 3. Finally, our results are summarised in Sect. 4.

\section{Code and settings}

\subsection{Code}

We carried out a 3D simulation with a code that solves the 3D gasdynamic equations, the Poisson equation for the gravitational field, and a rate equation for neutral hydrogen, including the transfer of ionising photons at the Lyman limit. The gas is initially atomic, and the models do not consider the photodissociation of molecular material because of the presence of a FUV radiation field. This code was described by Raga et al. (2008).

We modified the code of Raga et al. (2008) to include the "two temperature" equation of state described by Esquivel \& Raga (2007, hereafter E07). This equation of state assigns temperatures between $10 \mathrm{~K}$ (for neutral gas) and $10^{4} \mathrm{~K}$ (for gas with fully ionised $\mathrm{H}$ ) with a linear dependence on the $\mathrm{H}$ ionisation fraction. Therefore, instead of solving an energy equation with the appropriate heating and cooling terms (see Raga et al. 2008), we replace it with this two-temperature equation of state. We also included the self-gravity of the gas. We use a successive over relaxation (SOR) method to solve the Poisson equation for the gravitational potential, and then include the gravitational force in the momentum and energy equations. We do not include a treatment of the diffuse, ionising photon field.

\subsection{Settings}

The computational domain has a size of $(3.0,1.5,1.5) \times 10^{18} \mathrm{~cm}$ (along the $x-, y$-, and $z$-axes, respectively), which is resolved with a uniform grid of $256 \times 128 \times 128$ grid points. We impose transmision boundaries in the $x$-direction and periodic boundaries along the $y$ and $z$-directions. The periodic conditions are imposed in the gasdynamic equations, in Poisson's equation (for the gravitational field), and in the radiative transfer equations.

We start with an inhomogeneous density structure with a power-law power-spectrum index of $-11 / 3$ (i.e. $P[k] \propto k^{-11 / 3}$, where $k$ is the wave-number), as described in Esquivel et al. (2003). The initial density structure does not have any motion. To simulate the edge of an HII region, the computational domain is divided into two portions with a dividing line at $x=$ $4 \times 10^{17} \mathrm{~cm}$ from the left edge of the domain. The portion to the left is filled with an ionised medium (with a temperature of $10^{4} \mathrm{~K}$, and the portion to the right is filled with a neutral medium (with a temperature of $10 \mathrm{~K}$ ). The average density in the neutral medium is a factor of 100 higher than the one in the ionised medium, and the transition between the two (also in terms of temperature and ionisation fraction) follows a tanh profile with a width of $\sim 10$ pixels. The resulting neutral structure has a mass of $228 M_{\odot}$.

To calculate the gravitational field, we only consider the gravitational force resulting from the density perturbations. In other words, we subtract a density $\rho_{0}=3.51 \times 10^{-24} \mathrm{~g} \mathrm{~cm}^{-3}$ (corresponding to the lower density regions in the initial distribution of neutral material) from the density used in Poisson's equation. In this way, we avoid a generalized collapse of the dense slab structure that fills the computational domain. We also run a simulation in which the gravitational force was "turned off" to illustrate the effect of the self-gravity of the gas.

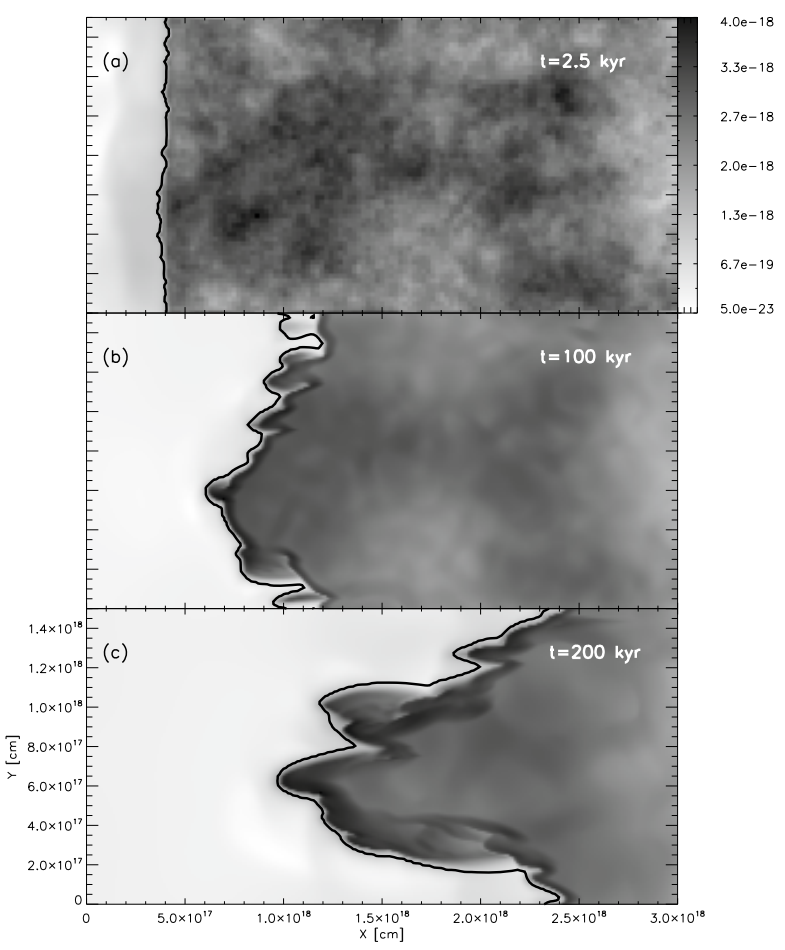

Fig. 1. Time evolution of the $x y$ mid-plane density stratification without self-gravity. The three frames are labeled with the corresponding elapsed times. The density stratifications are shown with the logarithmic greyscale given (in $\mathrm{g} \mathrm{cm}^{-3}$ ) by the top right bar. In the three frames, we also show the contour corresponding to an $\mathrm{H}$ ionisation fraction of $50 \%$, which indicates the position of the ionisation front. The $x$ and $y$-axes are labeled in $\mathrm{cm}$.

A plane-parallel ionising photon field $F_{0}=8.8 \times 10^{10} \mathrm{~cm}^{2} \mathrm{~s}^{-1}$ is incident on the computational domain along the $x$-axis. This photon flux corresponds to a star with an ionising photon rate $S_{*}=10^{48} \mathrm{~s}^{-1}$, located at a distance $D=9.5 \times 10^{17} \mathrm{~cm}$ from the edge of the computational domain in the $-x$ direction.

\section{Results}

We allowed the model to run from the initial conditions described in Sect. 2, to a $t=200 \mathrm{kyr}$ evolutionary time. Figure 1 shows the time evolution in the mid-plane density stratification without including the self gravity of the gas. Figure 2 shows the same simulation but adding the force that arises from self-gravity.

From both figures, it is evident that the ionisation front becomes highly corrugated with dense condensations at the tip of a number of protruding "fingers". At $t=100 \mathrm{kyr}$, the effect of selfgravity is only to produce denser condensations at the tip of the fingers. At $t=200 \mathrm{kyr}$, however, the density structures obtained without (Fig. 1) and with self-gravity (Fig. 2) are quite different. In the self-gravitating simulation, a dense, central structure (detached from the ionisation front and absent in the non-gravitating simulation) is produced.

Given the important differences found when including selfgravity, we present an analysis of clump formation only for the flow obtained from the self-gravitating simulation. Interestingly, if one repeats the analysis for the non-gravitating simulation, similar results are found (these results are not shown in the present paper).

To quantify the number of clumps produced, we calculate following E07 the number of spatially connected neutral 


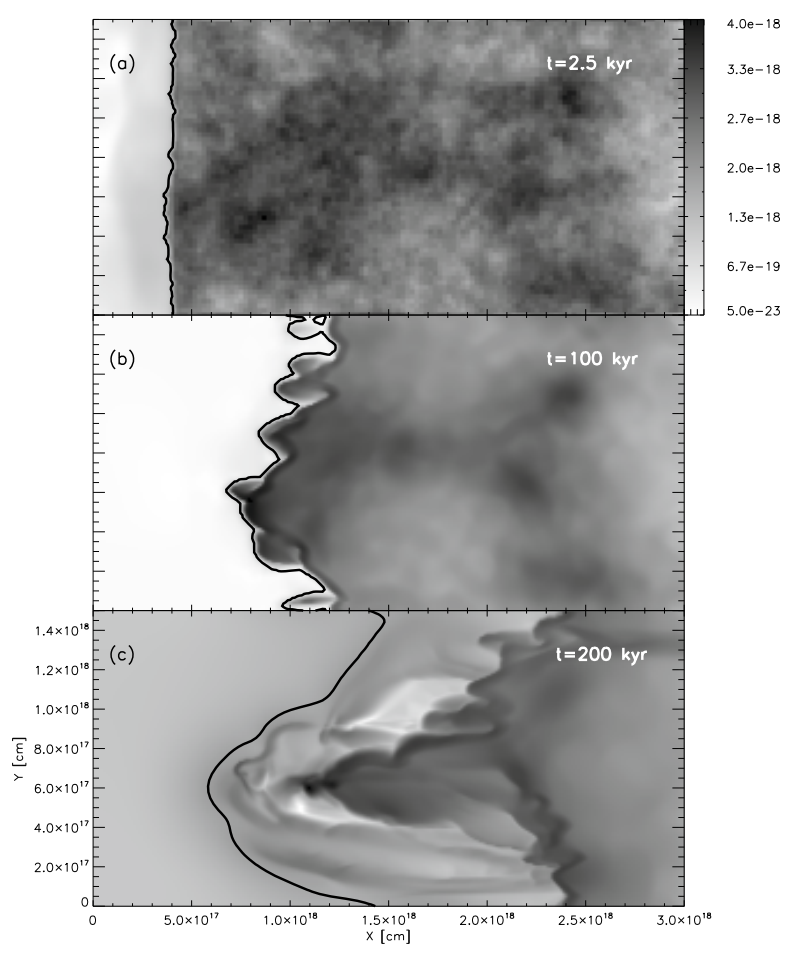

Fig. 2. Same as Fig. 1, but for the simulation that includes the self-gravity of the gas.

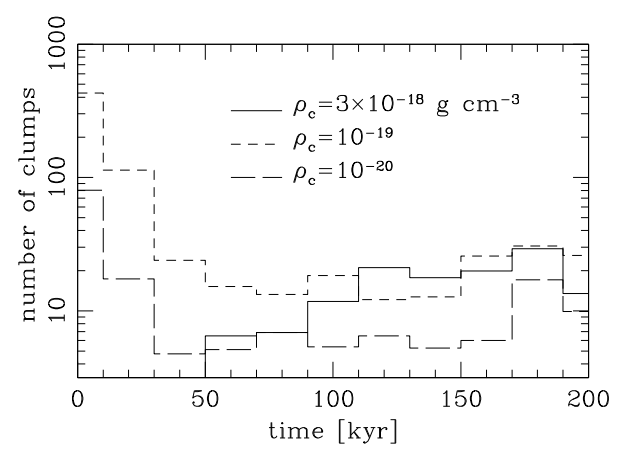

Fig. 3. Number of neutral clumps as a function of time, obtained for three different density cuttoffs. The results correspond to the simulation that includes self-gravity (see Fig. 2).

structures with densities above a specified cutoff density $\rho_{\mathrm{c}}$. In particular, we choose cutoff values of $\rho_{\mathrm{c}}=3 \times 10^{-18}, 10^{-19}$ and $10^{-20} \mathrm{~g} \mathrm{~cm}^{-3}$.

The number of clumps (fragments) obtained for different density cutoffs, is shown as a function of time in Fig. 3. To determine the number of clumps, we consider time intervals of $20 \mathrm{kyr}$ (corresponding to the width of the bins in the histograms of Fig. 3). We then calculate the number of clumps in 8 outputs within each of these time intervals, and then compute an average number of clumps for each time interval.

For the lowest cutoff density $\left(\rho_{\mathrm{c}}=10^{-20}\right)$, the initial density distribution has $\sim 80$ clumps, and the number of clumps first decreases with time, stabilizes at $\sim 5$ for $40<t<170 \mathrm{kyr}$, and then continues to increase a little at $t>170 \mathrm{kyr}$ (see Fig. 3). For the intermediate cutoff density $\left(\rho_{\mathrm{c}}=10^{-19}\right)$, the initial distribution has $\sim 500$ clumps and the number of clumps first decreases and then remains approximately constant as a function of time (with a value of $\sim 20$ ).

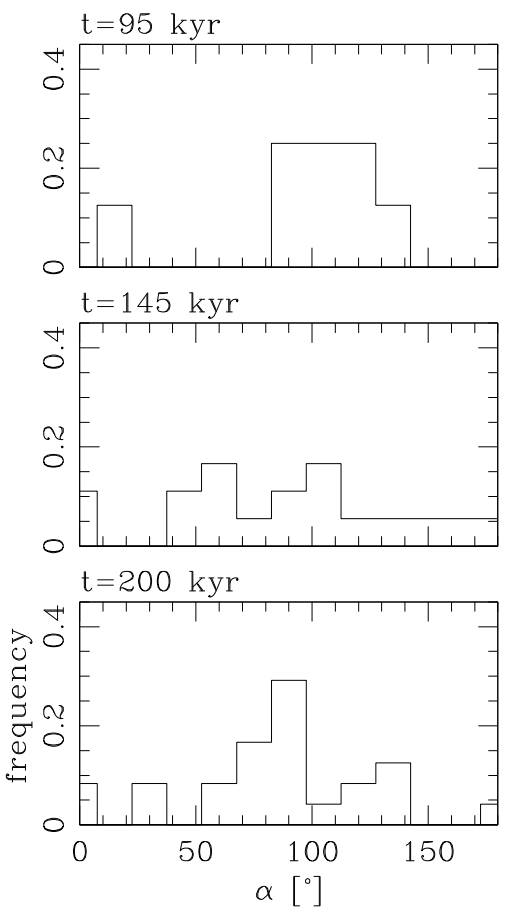

Fig. 4. Fraction of the clumps (obtained for a cutoff density $\rho_{\mathrm{c}}=$ $3 \times 10^{-18} \mathrm{~g} \mathrm{~cm}^{-3}$ ) with different orientations $\alpha$ (between the $x$-axis and the $x y$-projections of the angular momenta of the clumps). The panels are labeled with the elapsed time corresponding to the time-frames from which the three angular distributions were obtained. The results correspond to the simulation that includes self-gravity (see Fig. 2).

The initial density distribution has no clumps with densities above the highest chosen cutoff density, $\rho_{\mathrm{c}}=3 \times 10^{-18} \mathrm{~g} \mathrm{~cm}^{-3}$ (see above). Interconnected structures of sufficiently density only start to appear at $t \approx 30 \mathrm{kyr}$, and their number grows monotonically with time, stabilizing at a number of $\sim 20$ for $t>110 \mathrm{kyr}$ (see Fig. 3). For each of the detected clumps, we first compute the position of the centre of mass

$\boldsymbol{R}_{\mathrm{CM}}=\frac{1}{M} \int_{V} \rho \boldsymbol{r} \mathrm{d}^{3} \boldsymbol{x}$,

where $V$ is the contiguous volume of the clump and

$M=\int_{V} \rho \mathrm{d}^{3} \boldsymbol{x}$,

is its mass. We then compute the angular momentum with respect to the centre of mass of each clump

$\boldsymbol{L}=\frac{1}{M} \int \rho\left(\boldsymbol{r}-\boldsymbol{R}_{\mathrm{CM}}\right) \times \boldsymbol{v} \mathrm{d}^{3} \boldsymbol{x}$.

We assume that we observe the computed flow along the $z$-axis (i.e., that the $x y$-plane of the computational domain is parallel to the plane of the sky). The angle

$\alpha=\left|\arctan L_{Y} / L_{X}\right|$,

(with $\boldsymbol{L}$ given by Eq. (3)) then corresponds to the orientation angle (with respect to the direction of the ionising photon field) of the angular momentum of the clumps projected onto the plane of the sky. As discussed in Sect. 1, these directions correspond to the directions in which bipolar outflows will eventually be ejected when (and if) the clumps form star+accretion disk systems. 


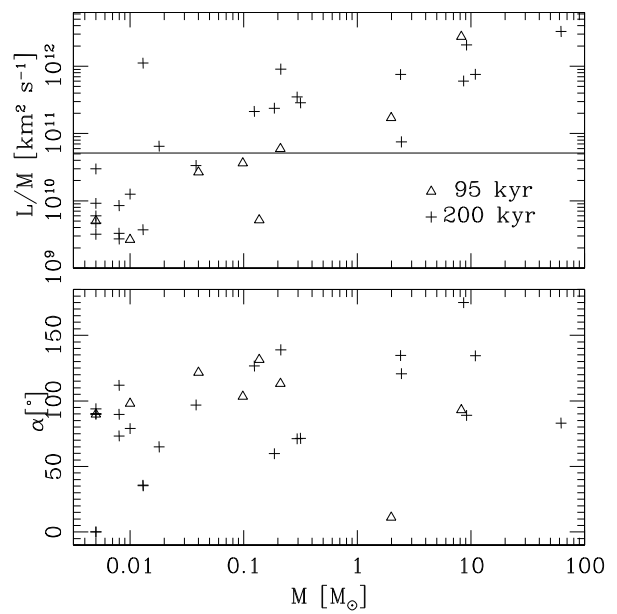

Fig. 5. The top panel shows the specific angular momentum $(L / M)$ of each clump as a function of the clump's mass. The black line shows the angular momentum associated with the outer orbit of an accretion disc radius of $r_{\mathrm{D}}=50 \mathrm{AU}$ around a $M=2 M_{\odot} \operatorname{star}\left(L / M \sim 5 \times 10^{10} \mathrm{~km}^{2} \mathrm{~s}^{-1}\right.$, see the text). The bottom panel shows the orientation $\alpha$ (between the $x$-axis and the $x y$-projection of the angular momentum) for each clump, as a function of its mass. The triangles show the clumps found for an elapsed time of $95 \mathrm{kyr}$ and the crosses for an elapsed time of $195 \mathrm{kyr}$. The clumps were obtained using a $\rho_{\mathrm{c}}=3 \times 10^{-18} \mathrm{~g} \mathrm{~cm}^{-3}$ density cutoff, using the results from the simulation of Fig. 2.

In Fig. 4, we show histograms indicating the fraction of clumps (obtained for a cutoff density $\rho_{\mathrm{c}}=3 \times 10^{-18} \mathrm{~g} \mathrm{~cm}^{-3}$ ) with different orientations $\alpha$, for the three different elapsed times $(t=$ 95,145 , and $200 \mathrm{kyr}$ ). For early times, we find that the $\alpha$ values of the clumps are randomly distributed (between $\sim 40$ and $180^{\circ}$ ). For $t=200 \mathrm{kyr}, \approx 36 \%$ of the clumps have $70^{\circ}<\alpha<100^{\circ}$, and more than $\approx 55 \%$ of the clumps have $60^{\circ}<\alpha<100^{\circ}$. From this result, we conclude that the dense clumps being formed have angular momenta preferentially aligned in directions perpendicular to the direction of the incident ionising photon field (which is parallel to the $x$-axis).

The bottom panel of Fig. 5 shows the projected orientation $\alpha$ of the angular momentum as a function of clump mass for all of the clumps obtained with the $\rho_{\mathrm{c}}=3 \times 10^{-18} \mathrm{~g} \mathrm{~cm}^{-3}$ cutoff density, for elapsed times $t=95$ and $200 \mathrm{kyr}$. We see that at $t=95 \mathrm{kyr}$ most of the clumps (triangles) have masses $M_{\mathrm{c}}<$ $0.3 M_{\odot}$ and angular momenta with all $\alpha$ orientations. For $t=$ $200 \mathrm{kyr}$ (crosses, see bottom panel of Fig. 5), we see that all of the clumps with $1 M_{\odot}<M_{\mathrm{c}}<60 M_{\odot}$ have angular momenta with orientation angles $70^{\circ}<\alpha<120^{\circ}$. The lower mass clumps (with $M_{\mathrm{c}}<0.3 M_{\odot}$ ) have angular momenta with more widely distributed orientations.

We computed the moduli of the specific angular momenta (i.e., the momentum per unit mass) of the clumps. The values of $L / M$ for $t=95$ and $195 \mathrm{kyr}$ are shown as a function of the clump mass $M$ in the top panel of Fig. 5 . For $t=95 \mathrm{kyr}$, we see that the clumps with $M_{\mathrm{c}}<0.3 M_{\odot}$ have $L / M<6 \times 10^{10} \mathrm{~km}^{2} \mathrm{~s}^{-1}$. The more massive clumps (with $M_{\mathrm{c}}>1 M_{\odot}$ ) have $L / M>6 \times$ $10^{10} \mathrm{~km}^{2} \mathrm{~s}^{-1}$. For $t=200 \mathrm{kyr}$, we see that the $M_{\mathrm{c}}<0.02 M_{\odot}$ clumps have $L / M<6 \times 10^{10} \mathrm{~km}^{2} \mathrm{~s}^{-1}$, while the clumps with $M_{\mathrm{c}}>0.02 M_{\odot}$ have $L / M>6 \times 10^{10} \mathrm{~km}^{2} \mathrm{~s}^{-1}$.

We now evaluate whether or not these specific angular momenta have values comparable to those observed in young star systems. Typical T Tauri stars have masses $M \approx 2 M_{\odot}$ and accretion disks with radii $r_{\mathrm{D}} \approx 50 \mathrm{AU}$. The outer Keplerian orbit of the disk then has a specific angular momentum $(L / M)_{\mathrm{D}} \approx$ $5 \times 10^{10} \mathrm{~km}^{2} \mathrm{~s}^{-1}$. This outer orbit is determined by the material
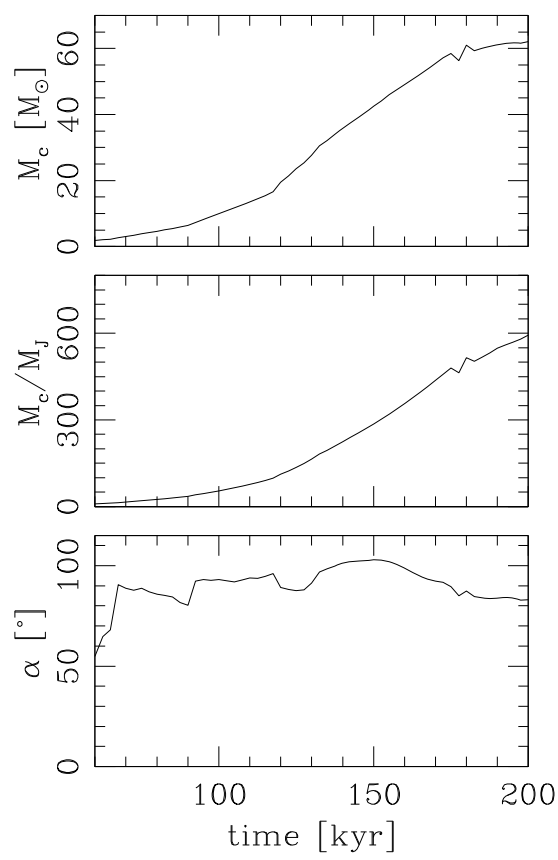

Fig. 6. Time evolution in the most massive, neutral clump obtained from the simulation with self-gravity (see Fig. 2). The top panel shows the mass, and the central panel shows the ratio of the clump mass to the Jeans mass as a function of time. The bottom panel shows the time evolution in orientation $\alpha$ of the angular momentum of this clump (where $\alpha$ is the angle between the $x$-axis and the $x y$-plane projection of the angular momentum).

of the highest angular momentum in the core from which the star+disk system was formed (see, e.g., Ulrich 1976).

This value of $(L / M)_{\mathrm{D}}$ is shown with a horizontal line in the top panel of Fig. 5. It is clear that many of the clumps formed in our simulation have specific angular momenta that are substantially higher than deduced from the radius of disks around $\mathrm{T}$ Tauri stars. From this, we conclude that the angular momenta of the clumps generated in our simulation are substantial.

A relevant question is whether the clumps obtained in our simulations are resolved well enough for the calculations of angular momenta to be meaningful. As an example, we consider the clumps found for the $\rho_{\mathrm{c}}=3 \times 10^{-18} \mathrm{~g} \mathrm{~cm}^{-3}$ cutoff density at time $t=200 \mathrm{kyr}$. The lower mass clumps (see Fig. 5) of $M \approx 0.01 M_{\odot}$ are resolved with $\sim 5$ grid points. The clumps of $M \approx 0.1 M_{\odot}$ are resolved with $\sim 50$ grid points. The clumps with $1<M<100 M_{\odot}$ are resolved with $\sim 500$ to 5000 grid points. Therefore, for clumps with $M>0.1 M_{\odot}$, the resolution of the internal structure of the clumps (with 50 grid points, corresponding to $\sim 4$ grid points along each axis) appears to be appropriate for obtaining a meaningful estimate of the angular momentum.

From the number of grid points $N_{\mathrm{c}}$ within the clumps, we can estimate the characteristic radii of the clumps to be $R_{\mathrm{c}}=$ $0.012 \mathrm{pc} \times N_{\mathrm{c}}^{1 / 3} / 2$, where $0.012 \mathrm{pc}$ is the resolution of the computational cells in our numerical simulation. From the values of $N_{\mathrm{c}}$ given in the previous paragraph, we then see that the clumps obtained from our simulation have characteristic radii $R_{\mathrm{c}} \approx 0.01,0.02,0.05$, and $0.1 \mathrm{pc}$, for clump masses of $M \approx 0.01$, $0.1,1$, and $100 M_{\odot}$, respectively.

Finally, we study the evolution in the most massive, neutral clump (detected with $\rho_{\mathrm{c}}=3 \times 10^{-18} \mathrm{~g} \mathrm{~cm}^{-3}$ ). As seen in Fig. 6 , this clump has a mass that grows monotonically from $0.6 M_{\odot}$ at $t=38 \mathrm{kyr}$, to $60 M_{\odot}$ at $t=200 \mathrm{kyr}$. The orientation angle $\alpha$ (on 


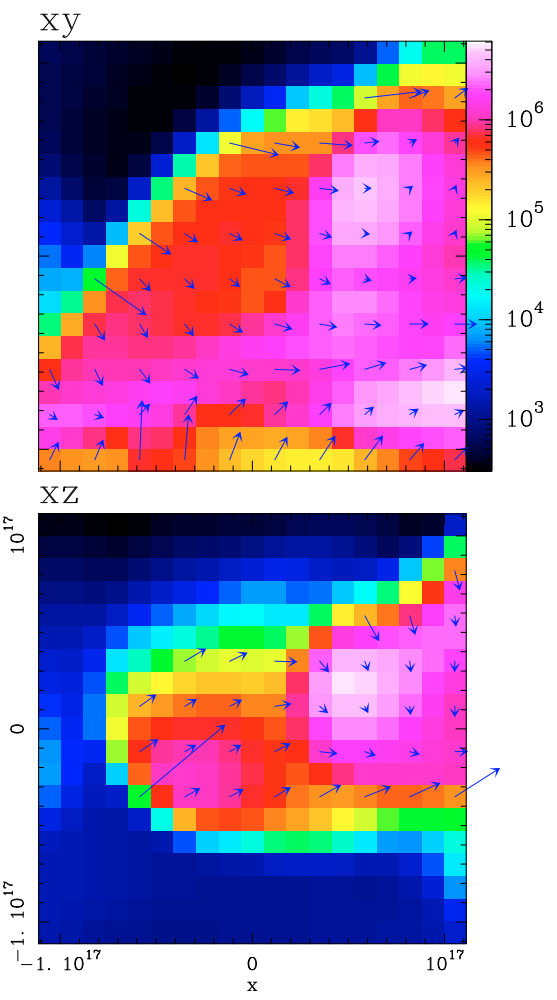

Fig. 7. Density stratification and flow field in a region around the centre of mass of the most massive clump at $t=200 \mathrm{kyr}$ (see Fig. 6). The top panel shows the flow on a $x y$-cut and the bottom panel the flow on a $x z$-cut. The origin of the coordinate system coincides with the centre of mass of the clump (obtained with a $\rho_{\mathrm{c}}=3 \times 10^{-19} \mathrm{~g} \mathrm{~cm}^{-3}$ density cutoff, which corresponds to a number density of $\left.1.4 \times 10^{5} \mathrm{~cm}^{-3}\right)$. The colour scale shows the logarithmic density distribution (given in $\mathrm{cm}^{-3}$ by the bar on the top right). The arrows show the flow velocities on the planes of the two cuts for densities $\rho>10^{-19} \mathrm{~g} \mathrm{~cm}^{-3}$. An arrow with a length equal to the separation between successive arrows corresponds to a flow velocity of $0.5 \mathrm{~km} \mathrm{~s}^{-1}$.

the plane of the sky) of its angular momentum stabilizes rapidly at $\alpha \approx 90^{\circ}$ for $t>70 \mathrm{kyr}$.

We compute the Jeans mass of this clump to

$M_{J}=\frac{1}{6} \pi \bar{\rho}\left(\frac{\pi c_{\mathrm{s}}^{2}}{G \bar{\rho}}\right)^{\frac{3}{2}}$

(see, e.g., E07) where $G$ is Newton's constant, $c_{\mathrm{s}}$ is the sound speed of the neutral medium (see Sect. 2), and $\bar{\rho}=M_{\mathrm{c}} / V^{1 / 3}$. We show the ratio $M_{\mathrm{c}} / M_{J}$ for the most massive clump as a function of time in the central panel of Fig. 6. It is clear that this clump is Jeans unstable for $\sim 160 \mathrm{kyr}$, which is a long enough timescale for the formation of a low mass star.

Figure 7 shows the density and flow velocity distributions in the $x y$ - and $x z$-planes, within a $\left(2 \times 10^{17} \mathrm{~cm}\right)^{2}$ region centred on the centre of mass of the most massive clump at $t=200 \mathrm{kyr}$. In the two cuts that are shown, we see that the region with densities higher than the $\rho_{\mathrm{c}}=3 \times 10^{-19} \mathrm{~g} \mathrm{~cm}^{-3}$ density cutoff (which corresponds to a number density of $1.4 \times 10^{5} \mathrm{~cm}^{-3}$ ) has a number of density maxima, none of which coincides with the centre of mass of the structure. The $x z$-plane (bottom panel) shows the velocity field that gives rise to the angular momentum of the clump.

\section{Conclusions}

We have presented the results of numerical simulations of a neutral structure with power-law density perturbations that is photoevaporated by an incident, plane-parallel, ionising photon field, with and without the self gravity of the gas. In this interaction, a number of dense, neutral clumps are produced. Our simulations are similar to those presented by Gritschneder et al. (2009). The main difference is that while they started their simulations in a medium with turbulent motions, our simulations begin in a stationary medium with density perturbations. In our simulations, the velocity field that develops is therefore mainly the result of the interaction with the ionising photon field. Defining clumps as contiguous structures above a cutoff density $\rho_{\mathrm{c}}$, we compute the statistics of the number of clumps as a function of elapsed time (for different values of $\rho_{\mathrm{c}}$ ). We then fix the cutoff density at $\rho_{\mathrm{c}}=3 \times 10^{-18} \mathrm{~g} \mathrm{~cm}^{-3}$, to focus on the denser clumps appearing at later elapsed times.

For these clumps, we compute the vector angular momenta, from which we obtain the direction of the rotation axes (projected on the plane of the sky) and the specific angular momenta. We find that as a function of evolutionary time we obtain orientations that are aligned increasingly perpendicular to the direction of the incident, ionising photon field.

For the most massive clump, we find that it has a mass that increases across the range $\approx 0.6-60 M_{\odot}$ (during the $t=38 \rightarrow$ $200 \mathrm{kyr}$ period), and that the orientation angle $\alpha$ of its angular momentum eventually stabilizes at $\alpha \approx 90^{\circ}$ (i.e., the direction perpendicular to the direction of the incident photon field). We use an estimate of the Jeans mass of the clump to show that it is Jeans unstable throughout the $t=38 \rightarrow 200 \mathrm{kyr}$ period. This timespan is long enough for a low mass star to form within the most massive clump. However, at the resolution of our simulation (with a grid spacing of $\approx 800 \mathrm{AU}$ ), we naturally do not succeed in form a star+disk system.

If we analyse our non-gravitating simulation (see Fig. 1), we obtain qualitatively similar results. Regardless of whether we consider the self-gravity of the gas or not, we produce clumps with angular momenta preferentially aligned perpendicular to the direction of the incident ionising photon field. Even though it is impossible to provide a full explanation of this alignment, it is possible to provide a qualitative explanation. During the interaction of the ionising photon field with a perturbed density structure, a corrugated ionisation front is produced. This ionisation front pushes a shock into the neutral gas, producing a sheared velocity field that is preferentially aligned with the $x$-axis (i.e., with the direction of the ionising photon field). This sheared velocity field eventually produces vortical motions that are perpendicular to both the $x$-axis and the direction of the shear. This motion is seen in the $x z$-plane velocity field around the most massive clump in the $t=200 \mathrm{kyr}$ frame shown in the bottom frame of Fig. 7.

We have shown that the dense clumps that form as the result of the photoevaporation of a dense, neutral structure in the ISM have angular momenta preferentially aligned in a direction perpendicular to the external ionising photon field. This result provides a natural explanation of the orientations observed in the HH 555 (Bally \& Reipurth 2003), HH 666 (Smith et al. 2004), and HH 333 (Rosado et al. 1999; Yusef-Zadeh et al. 2005) outflows, which emerge from elephant trunks in directions approximately perpendicular to the body of the trunks. Future observations of $\mathrm{HH}$ flows emerging from externally photoionised, neutral structures will show whether or not this kind of orientation is a general property of these outflows. 
We note again that we have simulated an ionisation front travelling into an initially steady, neutral medium with density perturbations. In this way, our simulations follow the dynamics produced by the propagating ionisation front and associated shock waves, which result in the production of clumps with angular momenta preferentially aligned perpendicular to the direction of the ionising photon source. In the real ISM, a medium with density perturbations also has associated motions, and an initial vorticity field that will influence the angular momenta of clumps that might form (e.g., in the interaction with an ionisation front). If the initial vorticity field is strong enough, it will probably hide the effect of the vorticity generated by the shocks associated with the ionisation front, and the angular momentum alignment effect described in this paper will not be present.

An evaluation of whether or not the vorticity generated by the ionisation front will be hidden by the initial vorticity field of the cloud (present before the perturbations associated with the approaching ionisation front) can be completed on the basis of observations of the rotation of dense clumps in molecular clouds. For example, Ohashi et al. (1997) observed the kinematics of a number of $\mathrm{NH}_{3}$ cores in IRAS $04169+2702$ and computed their specific angular momenta. They find that cores with radii in the $0.02 \rightarrow 0.1 \mathrm{pc}$ range have specific angular momenta $L / M \sim(0.3 \rightarrow 3) \times 10^{11} \mathrm{~km}^{2} \mathrm{~s}^{-1}$ (clumps with larger radii having specific angular momenta up to an order of magnitude higher for $\mathrm{a} \sim 1$ pc clump radius).

In our simulation, the clumps with radii in the $0.02 \rightarrow 0.1 \mathrm{pc}$ range (corresponding to clump masses in the $0.1 \rightarrow 100 M_{\odot}$ range, see Sect. 3), have angular momenta $L / M \sim(0.4 \rightarrow$ $20) \times 10^{11} \mathrm{~km}^{2} \mathrm{~s}^{-1}$ (see Fig. 5). Therefore, our clumps have angular momenta with values ranging from the lower $L / M$ values of the cores observed by Ohashi et al. (1997), up to a factor of $\sim 10$ times higher than the observed values. This result indicates that if the initial specific vorticity of the structure in the cloud were comparable to that of IRAS $04169+2702$, the passage of an ionisation front would generate clumps of considerably higher specific vorticity, and therefore the angular momentum alignment effect described in this paper would indeed be present (at least for the more massive, higher angular momentum clumps).

As a final point, we note that in the simulations presented in this paper we consider only the photoionisation of a neutral structure. In the case of the interaction of the radiation of an O star with a molecular cloud, it is unavoidable that the region outside the ionisation front will be affected by the FUV radiation from the star, which at least partially photodissociates the initially molecular material. Gorti \& Hollenbach (2002) computed models of the photodissociation of dense clumps, and concluded that clumps with central column densities $<2 \times 10^{22} \mathrm{~cm}^{-2}$ (for an assumed cold-to-dissociated gas sound speed ratio of $\sim 1 / 3$ ) will be rapidly photodissociated, and disappear as local density enhancements. In our simulations, the clumps that are produced have central column densities of $\sim(4,9,23,47) \times$ $10^{22} \mathrm{~cm}^{-2}$ for clump masses of $0.01,0.1,1$, and $100 M_{\odot}$, respectively (these central column densities are estimated by multiplying the clump radii given in Sect. 3 by the cutoff density of $\sim 1.5 \times 10^{6} \mathrm{~cm}^{-3}$ ). Therefore, in all cases the clumps have high enough column densities to avoid their dissipation by the incident FUV field.

From the results of Gorti \& Hollenbach (2002), we therefore conclude that the photodissociation caused by the FUV field will not destroy the clumps produced in our simulations. However, the early evolution of the flow (in which high density structures have not yet formed) might indeed be modified by the presence of a FUV field. It will therefore be interesting to carry out a future exploration of the formation of clumps within elephant trunks in the presence of both a photodissociating and a photoionising photon field.

Acknowledgements. We acknowledge support from the CONACyT grant 61547. V.L. acknowledges the CONACyT scholarship 194595 and Stu group. We thank an anonymous referee for helpful suggestions. We thank Malcolm Walmsley for pointing out that the observations of angular momenta of cores are relevant for the present work (giving rise to the four last paragraphs of Sect. 4).

\section{References}

Bally, J., \& Reipurth, B. 2003, AJ, 126, 893

Bally, J., Reipurth, B., \& Davis, C. J. 2007, Protostars and Planets V, ed. B. Reipurth, D. Jewitt, \& K. Keil (Univ. of Arizona Press), 215

Esquivel, A., \& Raga, A. C. 2007, MNRAS, 377, 383 (E07)

Esquivel, A., Lazarian, A., Pogosyan, D., \& Cho, J. 2003, MNRAS, 342, 325

Gahm, G. F., Carlqvist, P., Johansson, L. B., \& Nikolić, S. 2006, A\&A, 454, 201

Gorti, U., \& Hollenbach, D. 2002, ApJ, 573, 215

Gritschneder, M., Naab, T., Walch, S., Burkert, A., \& Heitsch, F. 2009, ApJ, 694, L26

Mellema, G., Arthur, S. J., Henney, W. J., et al. 2006, ApJ, 647, 397

Ohashi, N., Hayashi, M., Ho, P. T. P., et al. 1997, ApJ, 488, 317

Raga, A. C., Henney, W., Vasconcelos, J., et al. 2008, MNRAS, in press

Reach, W. T., Faied, D., Rho, J., et al. 2009, ApJ, 690, 683

Rosado, M., Esteban, C., Lefloch, B., Cernicharo, J., \& García López, R. J. 1999, AJ, 118, 2962

Smith, N., Bally, J., \& Brooks, K. 2003, AJ, 127, 2793

Ulrich, R. K. 1976, ApJ, 210, 377

Yusef-Zadeh, F., Biretta, J., \& Wardle, M. 2005, ApJ, 624, 246 